\title{
Management of lung cancer in the COVID-19 pandemic: a review
}

\author{
Fernando Maroto Piñeiro', Carmen Trinidad López², Javier de la Fuente Aguado \\ 'Department of Internal Medicine Hospital Povisa Ribera Salud Salamanca 5, Vigo 36211, Spain. \\ ${ }^{2}$ Radiology Service Hospital Povisa Ribera Salud Salamanca 5, Vigo 36211, Spain.
}

Correspondence to: Dr. Javier de la Fuente Aguado, Hospital Povisa Ribera Salud, Salamanca 5, Vigo 36211, Spain.

Email: jfuente@povisa.es

How to cite this article: Maroto Piñeiro F, Trinidad López C, de la Fuente Aguado J. Management of lung cancer in the COVID-19 pandemic: a review. J Cancer Metastasis Treat 2021;7:10. http://dx.doi.org/10.20517/2394-4722.2020.115

Received: 16 Oct 2020 First Decision: 25 Nov 2020 Revised: 10 Dec 2020 Accepted: 13 Jan 2021 Published: 7 Feb 2021

Academic Editors: Robert Kratzke, Lucio Miele Copy Editor: Xi-Jun Chen Production Editor: Yue-Yue Zhang

\begin{abstract}
Since its inception, the COVID-19 pandemic has affected health care as a whole. Cancer patients in general and those suffering from lung cancer in particular are a vulnerable group because of their many intrinsic characteristics and care needs. How SARS-CoV-2 (COVID-19) infection affects these patients regarding their risk of infection and outcome in this patient cohort is still to be determined. In this review, we tried to summarize our main concerns regarding COVID-19 in the context of cancer patients from a clinical and multidisciplinary approach. Different types of lung cancer treatments (chemotherapy, radiation therapy and immunotherapy) may also influence the risk of infection and condition the patient's risk of having a worse outcome. Lung cancer patients require frequent radiologic study follow-ups, which may be affected by COVID-19 pandemic. COVID-19 related incidental radiologic findings can appear in routinely scheduled radiology tests, which may be difficult to interpret. Also cancer treatment induced pneumonitis may have similar radiologic features similar to those in acute SARS-CoV-2 pneumonia and lead to a wrong diagnosis. The different health care needs, the requirement for continuous health care access and follow-ups, and the clinical traials in which this patient population might be enrrolled are all being affected by the current COVID-19 health crisis. The COVID-19 pandemic has put health care providers and institutions in difficult situations and obliged them to face challenging ethical scenarios. These issues, in turn, have also affected the psychological well-being of health care workers.
\end{abstract}

Keywords: COVID-19, cancer; ethics, CT thorax, lung cancer, cancer treatment, chemotherapy, immunotherapy

\footnotetext{
(@) $\left(\frac{1}{\infty}\right.$

(C) The Author(s) 2021. Open Access This article is licensed under a Creative Commons Attribution 4.0 International License (https://creativecommons.org/licenses/by/4.0/), which permits unrestricted use, sharing, adaptation, distribution and reproduction in any medium or format, for any purpose, even commercially, as long as you give appropriate credit to the original author(s) and the source, provide a link to the Creative Commons license, and indicate if changes were made.
}

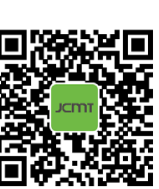




\section{INTRODUCTION}

The year 2020 was marked by the appearance of the current COVID-19 pandemic that has affected millions of people around the world and has challenged the health care systems of all countries.

COVID-19 is a systemic viral disease that specifically compromises the respiratory system and presents with different levels of severity, which depends mainly on the immune response of the host. As in any infectious disease, patients with cancer are a particularly vulnerable population against COVID-19, and their need for continuous care and regular contact with the hospital environment puts them at a risk of COVID-19 contagion. On the other hand, the degree of respiratory compromise due to the cancer itself is an additional factor of poor prognosis if they are infected. Also, chemotherapy and specific immunotherapeutic drugs may affect the immunological state of the patient and the outcome of a viral infection. The care of these patients in the current epidemiological situation represents a challenge for professionals and institutions, given the limited experience generated in the pandemic. In this article, we summarize the existing evidence in the literature on COVID-19 infection in patients with lung cancer and highlight aspects that we consider most relevant in this clinical setting.

\section{SARS COV-2}

SARS COV-2 is an RNA virus belonging to the family Coronaviridae. This group of viruses can cause infection in humans and animals with different levels of severity. They are usually seasonal, predominant in winter, and can affect a wide variety of animals ${ }^{[1]}$. In December 2019, a new coronavirus called severe acute respiratory syndrome coronavirus 2 (SARS-CoV-2) triggered an outbreak that caused a global pandemic, which we suffer to date.

The virus spread worldwide very fast. The main transmission medium is thought to be person-to-person respiratory transmission by respiratory droplets ${ }^{[2]}$ when close contact happens. Some environmental factors may have contributed to rapid spreading of SARS-CoV-2, for example Xie et al. ${ }^{[3]}$, described that low temperatures, especially those below $3{ }^{\circ} \mathrm{C}$ contributed to more successful viral transmission in a linear regression model estimated on the basis of the cases and climate conditions in 122 Chinese cities. Air pollution has also been assumed to be associated with higher transmission rates. $\mathrm{Coccia}^{[4]}$ published an article showing that cities with higher pollution rates and lower wind speeds had more COVID-19 cases in the first few months of the pandemic outbreak in Italy, and prevention strategies were also discussed in the $\operatorname{article}^{[4,5]}$. Other research findings also supported the association between SARS-CoV-2 and climate factors such as temperature, wind speed or solar radiation ${ }^{[6]}$ and air pollutants ${ }^{[7]}$. The most frequent symptoms of SARS-CoV-2 infection are fever, cough, dyspnoea, ageusia, and myalgia. Some patients with COVID-19 have serious complications including acute respiratory distress syndrome, severe pneumonia, acute kidney failure, septic shock, and neurological and thrombotic dysfunction ${ }^{[7]}$. According to data provided by the European Center for Disease Prevention and Control, up to $18 \%$ of patients admitted to hospitals require admission to an intensive care unit ${ }^{[8]}$.

There are many ongoing clinical trials that are investigating potential treatments for COVID-19. The controlled, open-label Randomized Evaluation of Covid-19 Therapy (RECOVERY) trial demonstrated the possible benefit of dexamethasone, especially the less need for invasive mechanical ventilation and the lower mortality of critically ill patients in need of oxygen supply ${ }^{[9]}$, because of which it was recommended by the National Institute of Health in the United States ${ }^{[10]}$. Clinical trials with Remdesivir have shown a decrease in recovery time in hospitalized patients with lower respiratory tract infections ${ }^{[11]}$ and a benefit in the clinical status of patients ${ }^{[12]}$. Remdesivir has been approved by the United States Food and Drug Administration for use in adult and pediatric patients 12 years of age and older and weighing at least 40 kilograms for the treatment of COVID-19 requiring hospitalization ${ }^{[13]}$. Hydroxychloroquine, holding 
potential anti-inflammatory and antiviral activity, did not show any benefit in the two most relevant clinical trials: WHO SOLIDARITY ${ }^{[14]}$ or RECOVERY ${ }^{[15]}$. Likewise, the trials carried out with lopinavir/ritonavir ${ }^{[16]}$ have not shown encouraging results either. There is modest evidence that the use of plasma from recovered COVID-19 patients could have benefits for infected patients ${ }^{[17]}$; however further studies are needed to draw conclusions in this regard.

Currently, there are several vaccines under development for COVID-19 based on different mechanisms that generate immunization (viral vectors, inactivated, attenuated viruses or recombinant proteins, among others $)^{[18]}$. Some of these vaccines are now approved by health institutions.

\section{CANCER AND THE COVID-19 PANDEMIC}

The immunosuppressed status of some cancer patients (either caused by the disease itself or by treatment) appears to increase their risk of infection to COVID-19 when compared to the general population. Although current evidence about the incidence of COVID-19 in cancer patients is limited, existing data seem to indicate that the incidence of infection in this group of patients is higher than in the general population. In a study carried out in a hospital in Madrid (Spain) between February 2020 and April 2020, a cumulative incidence of $4.2 \%$ was observed in patients admitted to the oncology service compared to a cumulative incidence of $0.63 \%{ }^{[19]}$. Likewise, in a study carried out in a hospital in China in early 2020, Yu et $a l .{ }^{[20]}$, reported a higher cumulative incidence of COVID-19 contraction in cancer patients compared to the rest of the general population that attended this hospital (0.79\% vs. $0.37 \%)$.

Immunosuppression can also make cancer patients more likely to present with complications SARS-Cov2 infection. Liang et al. ${ }^{[21]}$, reported that patients with neoplasms have a 3.5 times higher risk of serious infections that require mechanical ventilation and admission to the ICU, and associated with greater number of deaths, compared to patients without cancer. A history of cancer was also found to confer the highest risk of serious complications and correlate with worse COVID-19 outcomes. In particular, lung cancer patients were more likely to experience serious complications compared to patients with other neoplasm, although the most common type of oncologic disease in COVID-19 patients in this study was found to be lung neoplasm. The increased susceptibility of cancer patients to severe complications of COVID-19 can be caused by the neoplasm itself or the different kinds of treatments this patients receive.

Other studies also support the theory that the rate of complications and mortality in patients with both COVID-19 and cancer is higher. In a meta-analyses published by Giannakoulis et al. ${ }^{[22]}$, and Venkatesulu et al. ${ }^{[23]}$, the results seem to indicate that the prognosis and mortality of COVID-19 are indeed higher in cancer patients.

Some studies suggest that patients with advanced cancer or certain types of neoplasia are more likely to develop serious complications from COVID-19. Among the clinical factors associated with worse outcomes, Tian et al. ${ }^{[24]}$, found that advanced cancer with metastasis worsened the prognosis of patients with COVID-19 and neoplastic disease.

SARS-CoV-2 infection in patients with lung cancer tends to be more severe and has a worse prognosis, similar to hematologic neoplasms. In a multicenter study by Dai et al. ${ }^{[25]}$, only patients with stage IV metastatic cancer, haematological neoplasms, or lung cancer showed a worse evolution. Similar results were obtained in the study carried out by Jee et al.$^{[26]}$, in patients under active chemotherapy treatment, wherein its diagnosis of lung neoplasia was determined as a predictor of poor prognosis in COVID-19 infection, also confirmed by other authors ${ }^{[27,28]}$. 
The reason why patients with lung neoplasia have a worse evolution is not known, and it is probably multifactorial. Pro-inflammatory states appear to be associated with a worse prognosis in COVID-19 infection. In particular, levels of interleukin 6 (IL-6) appear to play an important role in SARS-CoV-2 infection $^{[29]}$. This hypothesis led to the use of IL-6 inhibitors (Tocilizumab ) as a possible treatment for COVID-19 ${ }^{[30]}$. The studies by Dai et al. ${ }^{[31]}$, and Luo et al.$^{[32]}$, demonstrated that IL-6 levels in patients with lung cancer and COVID-19 were higher than in SARS-CoV-2 infected patients without a neoplastic disease. However, we must not forget that lung cancer causes serious alterations in the lung parenchyma and is associated with high mortality ${ }^{[33]}$, and it is described as a risk factor for worse prognosis in pneumonia due to influenza and other viruses ${ }^{[34]}$.

We must be cautious when concluding facts about COVID-19 prognosis and outcome on cancer patients, given that different treatments or cancer types may cause different responses to SARS-CoV-2 infection, and many variables and confounders may play different roles. For example, patients suffering from lymphoid malignancies with severe lymphopenia may have a poorer response to viral infection, while chemotherapy induced neutropenic patients may present an attenuated inflammatory reaction to the virus ${ }^{[35]}$.

\section{ONCOLOGICAL TREATMENT AND ACTIVE SARS-COV-2 INFECTION}

The evidence regarding cancer treatment and COVID-19 infection is mixed and contradictory.

A priori, we could expect that chemotherapy, being an immunosuppressive factor, could increase the risk of infection or lead to a worse prognosis in patients with COVID-19. Some studies support this hypothesis: Liang et al. ${ }^{[21]}$, reported that patients who had received chemotherapy or surgery in the 30 days before presenting with the SARS-CoV-2 infection had an increased risk of severe complications. Zhang et al.$^{[36]}$, reported that receiving cancer treatment within 14 days of being diagnosed with COVID-19 could increase the probability of a suffering a serious complication.

In contrast, an international multicenter study that recruited patients with both COVID-19 and oncological disease from Spain, Canada and the United States ${ }^{[37]}$, did not show a worse evolution of the infection among those patients who received a specific cancer treatment in the following 30 days. Similarly, in a recently published cohort study, Lee et al. ${ }^{[38]}$ found no relationship between the administration of cancer treatment in the previous days and mortality from COVID-19, while age and comorbidities were associated with a worse outcome.

Currently, the American Society of Clinical Oncology (ASCO) ${ }^{[39]}$ and the Spanish Society of Medical Oncology ${ }^{[40]}$ recommend suspending chemotherapy treatment in case of COVID-19 infection and assessing its continuation once the infection has passed.

There is concern about immunotherapy treatment in lung cancer, since the effects on the immune response to SARS-CoV-2 infection are not exactly known ${ }^{[41]}$. The latest recommendations from the American Society of Oncology advise evaluating each case individually and with caution ${ }^{[42]}$.

Target therapies for lung cancer have revolutionized clinical practice in the last few years and have improved patient's survivall ${ }^{[43]}$. In the first months of the pandemic, in the absence of reliable information, clinical reasoning and individualized decision-making have taken on special value. That is why the individual experiences of each professional are important and help in making clinical decisions, thus communication is of vital importance. For example, Zhang et al. ${ }^{[4]}$, reported the case of a Chinese patient with lung cancer who was positive for COVID-19 and was receiving immunotherapy with Osimertinib. The patient received treatment with lopinavir and ritonavir for SARS-CoV-2 infection and showed clinical improvements within 2 weeks, while also presenting with a negative nasopharyngeal exudate PCR test. In 
this case, the clinical condition and functional status of the patient allowed the continuation of his cancer treatment despite the diagnosis of COVID-19.

In the case of radiotherapy, we have not found consistent evidence regarding its effect on the evolution and prognosis of COVID-19. However, the latest ASCO recommendations ${ }^{[39]}$ advise suspending treatment during active infection. On the other hand, the NICE guidelines on the administration of radiotherapy and COVID-19 ${ }^{[45]}$ state that an active SARS-CoV-2 infection should not be considered as the only factor when evaluating the administration of radiotherapy and that each individualized patient situation should be taken into account. We encourage readers to frequently consult society clinical practice guidelines for advice, given the rapidly changing scientific evidence.

\section{DIAGNOSIS AND IMAGE FOLLOW-UP WITH IMAGING IN COVID-19 AND LUNG CANCER PATIENTS: KEY ASPECTS}

In patients with lung cancer, chest computed tomography (CT) is the imaging technique of choice for the diagnosis, staging, and follow-up, as well as for planning treatment with radiotherapy ${ }^{[46]}$. CT has a very relevant role in COVID-19, both in the diagnosis and in the screening of serious complications such as pulmonary thromboembolism, bacterial or fungal infection ${ }^{[4]]}$.

CT has been reported to have a sensitivity of up to $97 \%$, which is significantly higher than RT-PCR in the diagnosis of COVID-19. In a study with 1014 patients from Wuhan in which both techniques were compared, positive confirmations were found in $88 \%$ with CT and $59 \%$ with RT-PCR and up to $93 \%$ of the patients were considered positive on CT before confirmation by RT-PCR test ${ }^{[48]}$. However, the specificity of CT was only $25 \%$ to $33 \%^{[49]}$.

Therefore, CT is a useful imaging technique, especially in the initial phases of infection when the RT-PCR is negative, but there is a high clinical and epidemiological suspicion when the follow-up CT of cancer patients not suspected of having an infection incidentally shows the typical radiological alterations of COVID-19.

The most frequent findings of COVID-19 infection on chest CT include the presence of ground glass opacities or patchy consolidations, which are associated or not with linear images due to thickening of the septa, peripherally located, bilaterally or multi-lobular; the presence of a diffuse alveolar pattern; or areas of organizing pneumonia ${ }^{[50]}$. When these findings are observed in a situation with a high prevalence of the disease, the diagnosis of COVID-19 infection is highly probable, and a clinical and epidemiological investigation must be carried out to rule it out.

In patients who have received radiotherapy weeks or months before the SARS-CoV-2 infection, a differential diagnosis must be made with radiotherapy pneumonitis (RP) given that it's clinical manifestations are similar to those of COVID: progressive dyspnoea and cough, with or without fever.

It is important to know the field of radiation therapy and the time elapsed since the end of treatment. Ground glass lesions and patchy consolidations appear on CT in the early stages of RP and are indistinguishable from COVID-19 infection. COVID-19 should be suspected when the lesions are bilateral and appear outside the radiation field ${ }^{[51]} .2$ to 24 months after the last radiation therapy dose, signs of fibrosis appear including traction bronchiectasis and loss of volume, and the presence of new-onset lesions gives rise to complex radiological patterns in which it is difficult to identify typical signs of COVID-19 infection. 
In patients treated with immunotherapy, pneumonitis may appear. In these cases, chest CT usually shows ground-glass opacities, consolidations, and mixed multifocal lesions, predominantly in the lower lobes. The most frequent radiological pattern (following the ATS/ERS classification) is that of an organizing pneumonia in up to $65 \%-70 \%$ of cases, but other patterns can be seen, including non-specific interstitial pneumonia, hypersensitivity pneumonitis, or interstitial acute pneumonia (diffuse alveolar damage or respiratory distress syndrome $)^{[52]}$. Both the pattern of organizing pneumonia and diffuse alveolar damage are indistinguishable from COVID-19 infection and in these cases, it is important to know the time of onset of the symptoms ${ }^{[53]}$.

Development of technology, artificial intelligence and deep machine learning may provide a useful tool in the future for COVID-19 and lung cancer radiological diagnosis, as it has shown to improve the diagnostic efficiency of lung cancer ${ }^{[54]}$.

\section{ACCESS TO HEALTH CARE FOR PATIENTS WITH LUNG CANCER DURING THE COVID-19 PANDEMIC: INITIATIVES IN HEALTH INSTITUTIONS}

The health crisis situation due to COVID-19 has caused hospitals and health care systems across different regions of the world to recommend delaying and reducing outpatient follow-ups to minimize the exposure to SARS-CoV-2. Patients with cancer in general and with lung neoplasm in particular require periodic follow-ups and have close contact with the hospital environment. In addition, the complications derived from cancer treatments and the underlying disease itself often need hospitalization.

According to a recent study that used a large medical claim database in the United States ${ }^{[55]}$, cancer-related procedures (screening, biopsies, therapy) significantly dropped during the year 2020 when compared to the previous year 2019. Specifically, lung biopsies dropped by 58\% and 47\% during April 2020 and July 2020, respectively; lung cancer screening dropped by $75 \%$ in April 2020 and outpatient visits dropped by $74 \%$ in April 2020.

Regarding lung cancer diagnosis, the $\mathrm{ASCO}^{[56]}$ recommends strategies such as prioritizing transthoracic biopsies over transbronchial techniques in order to minimize aerosol generating procedures, and avoiding invasive approaches such as endobronchial ultrasound for staging, when possible.

The scheduled follow-ups of cancer patients are carried out based on recommendations that often leave a margin period to the physician's criteria. In a survey conducted by the Collegio Italiano dei Primari Oncologi Medici ${ }^{[57]}$ on 122 oncologists, it was corroborated that the delay in non-urgent outpatient visits was a widely adopted proactive measure in Italy. Similarly, Wang et al. ${ }^{[58]}$, published their experience of a hospital setting in the province of Sichuan (China) in which outpatient visits were reduced by $71 \%$ and there were no cases of COVID-19. Although it seems logical to think that reducing the frequency of visits and patient contact with the hospital environment are beneficial measures, long-term studies will be necessary to evaluate the impact that these protocols will have on the prognosis and survival of cancer patients.

When deferring follow-ups and scheduled radiologic tests in lung cancer patients, communication is key, and patient preferences, worries and risk perceptions must be taken into consideration. Evidence-based and objective information must be given to patients, and decision-making must be individualized. This aspect was highlighted by the authors of a recently published paper ${ }^{[59]}$, which discusess the conclusions of an expert panel on lung nodule management during the COVID-19 pandemic. 
Other tools to reduce overcrowding and potential exposure to COVID-19 in outpatient clinics are virtual health services such as teleconsultation or telemonitoring, especially for routine checks or refilling prescriptions. In this sense, the latest ASCO recommendations advise the use of telemedicine systems when possible for the follow-up of cancer patients ${ }^{[39]}$. Lonergan et al. ${ }^{[60]}$, published their experience with an initiative related to telemedicine and oncology in an oncology center at the University of California (United States) in which telematic consultations increased from 2,284 to 12,946 in a period of 11 weeks, immediately before the start of the pandemic. This initiative made it possible to continue maintaining access to health care for these patients at all times. However, telemedicine involves a series of inconveniences such as communication barriers (deafness, language), legal problems related to confidentiality and data protection, and difficulties in handling computer or electronic equipment due to cognitive problems, advanced age, or lack of internet access, among others ${ }^{[61,62]}$.

Home health care systems have shown benefits in the treatment of cancer patients, especially in the case of palliative care, showing an increase in the quality of life of patients ${ }^{[63]}$ and more cost-effectiveness of care ${ }^{[64]}$. The epidemiological situation derived from COVID-19 has further increased interest in home care, since the management of SARS-CoV-2 infection at home has been a useful tool in many countries. In Spain, home care in mild cases of COVID-19 is seen as a fundamental pillar of care by the Ministry of Health, Social Affairs and Equality ${ }^{[65]}$. This type of care is of particular interest, for example, in the case of cancer patients under palliative care, in which intensive therapeutic measures have been ruled out and the patient's greater comfort is sought in their family environment, reducing anxiety and other factors associated with hospitalization $^{[66]}$.

In some countries and health systems, the administration of chemotherapy by home hospitalization units has previously been carried out with good results ${ }^{[67]}$. Candidate patients are, in addition to those who receive oral chemotherapy, patients who receive intravenous chemotherapy with low intensity regimens. Other factors to consider are clinical stability, need for storage and processing of drugs, family support, and capacity of caregivers to be educated in different measures including follow-ups, risks for adverse reactions and anaphylaxis ${ }^{[68]}$. In a project carried out in 2019 in the United Kingdom in which the administration of home chemotherapy was implemented in patients with myelodysplastic syndrome ${ }^{[6]]}$, satisfaction surveys showed that $100 \%$ of the patients had noticed a positive change in their experience treatment. Nevertheless, the position of some oncology societies such as ASCO does not advise these modalities of treatment in general, except for selected patients ${ }^{[39]}$.

In general, cancer treatment is recommended in oncology patients who do not present with an active SARS$\mathrm{CoV}-2$ infection. Delaying the treatment of metastatic disease causes a worsening of one's functional status, increases the number of admissions for palliative support, and causes disease progression ${ }^{[70-72]}$. Kutikov et al. ${ }^{[73]}$, have proposed specific recommendations that can be used to guide the decision-making process when delaying or continuing cancer treatment during the COVID-19 pandemic. These recommendations are mainly based on categorizing patients at low, medium or high risk of disease progression depending on the type and extension of the disease.

Some authors have pointed out that the greatest danger for cancer patients in the current pandemic situation is the difficult access to the health care system and resources, which sometimes implies greater complications and diagnostic delays ${ }^{[7]}$. This situation is also being detected in other areas of medicine. For example, in Spain there has been a marked decrease in the number of acute coronary syndromes that receive hospital care coinciding with the times of highest incidence of COVID-19. This finding has been related with the population's fear of coming into contact with the hospital environment ${ }^{[75]}$. 
For all these reasons, it is essential that, despite the current situation, we should give out best efforts to provide excellent care to our patients from a multidisciplinary approach.

\section{GUIDELINES AND CLINICAL RESOURCES FOR THE MANAGEMENT OF ONCOLOGIC PATIENTS DURING THE COVID-19 PANDEMIC}

In the current epidemiological context, different clinical resources published by different institutions have emerged in order to guide patients, professionals and health institutions to take appropriate measures for the continuity and excellence of care. Many of these resources are in the form of web pages of different medical societies and patient associations in different countries.

The British Columbia Cancer Center has posted on its website a dossier ${ }^{[76]}$ that includes many frequently asked questions from cancer patients and provides appropriate instructions on necessary precautions regarding the detection of signs and symptoms of COVID-19; social distancing; personal protective equipment; and hospital visits. Additionally, the Cancer Council of Australia has published a multilingual cancer patient briefing that includes up-to-date information on COVID-1 $9^{[77]}$.

Recently, the National Institute for Excellence in Health and Care (NICE) published a clinical guideline regarding recommendations for oncologic treatments in the present epidemiological context ${ }^{[78]}$, which can help health providers with the latest evidence regarding this topic.

The European Society for Medical Oncology has developed an updated online clinical resource page to help collect all cancer-related COVID-19 clinical issues and the latest available evidence to provide the best care possible to cancer patients ${ }^{[79]}$.

The Royal College of Radiologists has also recently established a clinical resource that includes guidelines on the management of many cancers, including lung neoplasm. during the COVID-19 pandemic. These guidelines give detailed recommendations on radiation therapy treatment, including radiation therapy, administered doses, and when to continue, delay, or interrupt treatment ${ }^{[80]}$.

\section{ETHICAL ASPECTS AND DECISION MAKING IN PATIENTS WITH CANCER AND COVID-19}

During the COVID-19 pandemic, many health systems have faced the challenge of an avalanche of patients in emergency medical services, hospitals, and intensive care units in the context of high demand for pharmaceuticals, shortage of personal protective equipment, and limitation on medical supplies. Physicians have had to make very tough and difficult decisions to optimize the use of advanced therapeutic options, such as invasive mechanical ventilation, based on many factors, including age, co-existing conditions, or prognosis. Unfortunately, this situation is not new, and has its historical precedents as reflected in a recently published editorial ${ }^{[81]}$, wherein Mannelli reflects on the scarcity of resources and the difficult decisions that many health care professionals had to deal with in the initial stages of the pandemic.

Patients with advanced-stage lung cancer have a poor prognosis per se, so oncologists and multidisciplinary care teams should discuss with patients the measures to take if their clinical situation worsens. Generally, we try to respect the wishes of patients when deciding whether they want to receive invasive mechanical ventilation and advanced life support treatments if necessary, providing them with information and resources about palliative care to help them decide. It is important to discuss these issues in advance, to be able to take the appropriate clinical action when the patient is not able to decide, thereby respecting their previous decisions ${ }^{[82]}$. The Ethics Committee of the American College of Surgeons has developed a guideline to assist health care professionals in the decision-making process regarding the allocation of resources in the present epidemiological context ${ }^{[83]}$. 
As suggested by Sterpetti ${ }^{[84]}$, based on the difficult situations in Italy during the first months of 2020 , it is of great importance to predict the need for resources (ICU, beds, specialized facilities and staff), and to organize them efficiently in order to face the most critical moments of the pandemic.

Clinical judgment is essential to determine whether cancer therapy should be continued or discontinued in patients with COVID-19. Health care providers caring for oncology patients are encouraged to review the latest literature related to cancer and COVID-19 to provide evidence-based and individualized care. There is an urgent need for well-designed studies to identify the long-term clinical consequences of either continuing or terminating specific treatments in oncology and haematology settings.

\section{ONCOLOGICAL CLINICAL TRIALS DURING THE PANDEMIC}

Clinical trials with new therapies constitute the basic pillar of clinical research. Like all aspects of clinical practice, clinical trials have been influenced by the COVID-19 pandemic. The new situation raises new and difficult questions, since it is unknown in what way the administration of these experimental treatments will influence the participants of clinical trials under the COVID-19 pandemic situation. In addition, trial protocols require frequent visits by patients to health care settings, which increases the chances of exposure to COVID-19. Doherty et al ${ }^{[85]}$, analyzed the aspects related to the adaptation of clinical trials to the new epidemiological situation, pointing out that many governments have made the legislation more flexible so that the more relaxed protocols can allow trials to continue. They also point out the need to prioritize the primary objectives of the trials and give less importance to the secondary ones, thus reducing the need for controls and subsequent contact with the hospital or health setting environment.

Another aspect to take into account in this regard is to avoid the abandonment of patients who adhere to clinical trial protocols and to facilitate the contact and availability of professionals, as indicated by Shuman and Pentz ${ }^{[86]}$. For such patients, it is essential that we adapt and make use of resources such as telemedicine or home care.

Despite the current situation, we must try to keep clinical research ongoing, since the prognosis and evolution of many patients will depend on it, and as professionals, it is in our hands to take on the challenge of adapting to the new situation.

\section{PSYCHOLOGICAL CARE FOR HEALTH CARE PROVIDERS}

The situation experienced during the COVID-19 pandemic has subjected health care professionals to stressful situations that have been shown to increase their anxiety levels. In a survey conducted in China ${ }^{[87]}$ medical health workers showed higher anxiety and psychosocial problems than non-medical health workers. Most of the studies carried out in this line have reached similar conclusions ${ }^{[88]}$. This situation may be even more worrisome among professionals affected by the burnout syndrome, which is increasingly frequent among physicians ${ }^{[89,90]}$.

In the case of cancer patients, professionals must face the greater challenges and ethical dilemmas that we have previously mentioned. For all this, the need to provide psychological support for professionals is evident, thus also preventing clinical errors derived from mental fatigue and stress. There are several published initiatives in the literature that have been implemented to improve the mental health of health care workers in the context of COVID-19 $9^{[91-93]}$.

\section{CONCLUSIONS}

The COVID-19 pandemic has had a great impact on the health care systems of most countries. Cancer patients, and especially those who suffer from lung cancer, when infected with SARS COV2, have a worse 
prognosis than the general population, either due to their own disease or due to the treatments they receive. The current epidemiological situation represents a challenge for professionals and institutions, especially when we take into account the scarce and limited evidence generated in the pandemic. Decisionmaking should be individualized based on the need for care and the risk of exposure to SARS-CoV-2, always considering the wishes and preferences of patients, as well as the ethical aspects.

When considering future perspectives, major efforts and coordination must be undertaken by health and political authorities and institutions to improve the fight against COVID-19 ${ }^{[94]}$. As in the case of target therapies for lung cancer and other fields of medicine, molecular biology may play a key role in the discovery of new treatments and vaccines for SARS-CoV-2 ${ }^{[95]}$. Technology, including telemedicine, artificial intelligence and may play a major role in the management of the actual crisis, as well as the health care and diagnosis of lung cancer patients.

In this work, we attempted to summarize the main evidence and topics linking COVID-19 and lung cancer, while also recognizing that this is not a systematic review of the literature nor a meta-analysis. We consider this to be the main limitation of our study, as there may be published literature or important topics overlooked in this review.

\section{DECLARATIONS}

\section{Authors' contributions}

Participated in the bibliographic search, review and selection of articles included in the review, design and structure of the work and writing: Maroto Piñeiro F, de la Fuente Aguado J

Participated in the selection, revision and writing of the articles related to the radiological aspects described in the article: Trinidad López C

\section{Availability of data and materials}

Not applicable.

\section{Financial support and sponsorship}

None.

\section{Conflicts of interest}

All authors declared that there are no conflicts of interest.

\section{Ethical approval and consent to participate}

Not applicable.

\section{Consent for publication}

Not applicable.

\section{Copyright}

(c) The Author(s) 2021.

\section{REFERENCES}

1. McIntosh K, Pearlman S. Coronavirus, including severe acute respiratory syndrome and Middle East respiratory syndrome. In John E. Bennett, Raphael Dolin, Martin J. Blaser, editors. Mandell, Douglas, And Bennett's principles and practice of infectious diseases. Philadelphia: Elsevier/Saunders; 2015. pp. 2030-8.

2. Mcintosh K. Coronavirus disease 2019 (COVID-19): Clinical features. UptoDate. [Last accesses on 2 Feb 2021]

3. Xie J, Zhu Y. Association between ambient temperature and COVID-19 infection in 122 cities from China. Sci Total Environ 2020;724:138201. 
4. Coccia M. Factors determining the diffusion of COVID-19 and suggested strategy to prevent future accelerated viral infectivity similar to COVID. Sci Total Environ 2020;729:138474.

5. Coccia M. An index to quantify environmental risk of exposure to future epidemics of the COVID-19 and similar viral agents: Theory and practice. Environ Res 2020;191:110155.

6. Rosario DKA, Mutz YS, Bernardes PC, Conte-Junior CA. Relationship between COVID-19 and weather: case study in a tropical country. Int J Hyg Environ Health 2020;229:113587.

7. Bashir MF, Ma BJ, Bilal, et al. Correlation between environmental pollution indicators and COVID-19 pandemic: A brief study in Californian context. Environ Res 2020;187:109652.

8. International Severe Acute Respiratory and Emerging Infections Consortium (ISARIC). COVID-19 Report: 19 May 2020 [Internet]: ISARIC; 2020. Available from: https://media.tghn.org/medialibrary/2020/05/ISARIC_Data_Platform_COVID-19_Report_19MAY20. pdf. [Last accesses on 2 Feb 2021]

9. RECOVERY Collaborative Group; Horby P, Lim WS, Emberson JR, et al. Dexamethasone in Hospitalized Patients with Covid-19Preliminary Report. N Engl J Med 2020;17:NEJMoa2021436.

10. National Institutes of Health (NIH). The National Institutes of Health COVID-19 Treatment Guidelines Panel Provides Recommendations for Dexamethasone in Patients with COVID-19 [Internet]. NIH; 2020 [updated 25 June 2020; cited 29 June 2020]. Available from: https:// www.covid19treatmentguidelines.nih.gov/dexamethasone/ [Last accesses on 2 Feb 2021]

11. Beigel JH, Tomashek KM, Dodd LE, et al. Remdesivir for the treatment of Covid-19 - final report. N Engl J Med 2020;383:1813-26.

12. Spinner CD, Gottlieb RL, Criner GJ, et al. Effect of Remdesivir vs standard care on clinical status at 11 days in patients with moderate COVID-19: a randomized clinical trial. JAMA 2020;324:1048-57.

13. Fda.com (Internet). United Stated Food and Drug Administration (FDA) c2020 (cited Dec 6th 2020). Available from: https://www.fda. gov/news-events/press-announcements/fda-approves-first-treatment-covid-19

14. World Health Organization (WHO). "Solidarity" clinical trial for COVID-19 treatments. Geneva: WHO; 2020 [cited 22 July 2020 ]. Available from: https://www.who.int/emergencies/diseases/novel-coronavirus-2019/global-research-on-novel-coronavirus-2019-ncov/ solidarity-clinical-trial-for-covid-19-treatments. [Last accesses on 2 Feb 2021]

15. Randomised Evaluation of COVid-19 Therapy (RECOVERY) Trial. No clinical benefit from use of hydroxychloroquine inhospitalised patients with COVID-19: Nuffield Department of Population Health University of Oxford; 2020 [updated 5 June 2020]. Available from: https://www.recoverytrial.net/news/statement-from-the-chief-investigators-of-the-randomised-evaluation-of-covid-19-therapy-recoverytrial-on-hydroxychloroquine-5-june-2020-no-clinical-benefit-from-use-of-hydroxychloroquine-in-hospitalised-patients-with-covid-19. [Last accesses on 2 Feb 2021]

16. Cao B, Wang Y, Wen D, et al. A trial of Lopinavir-Ritonavir in adults hospitalized with severe Covid-19. N Engl J Med 2020;382:1787-99.

17. European Commission-Directorate-General For Health And Food Safety. An EU programme of COVID-19 convalescent plasma collection and transfusion. Guidance on collection, testing, processing, storage, distribution and monitored use. 2020. Available from: https://ec.europa.eu/health/sites/health/files/blood_tissues_organs/docs/guidance_plasma_covid19_en.pdf. [Last accesses on 2 Feb 2021]

18. Draft landscape of COVID-19 candidate vaccines. Available from: https://www.who.int/publications/m/item/draft-landscape-of-covid-19candidate-vaccines [Last accesses on 2 Feb 2021]

19. Rogado J, Obispo B, Pangua C, et al. Covid-19 transmission, outcome and associated risk factors in cancer patients at the first month of the pandemic in a Spanish hospital in Madrid. Clin Transl Oncol 2020;22:2364-8.

20. Yu J, Ouyang W, Chua MLK, Xie C. SARS-CoV-2 transmission in patients with cancer at a tertiary care hospital in Wuhan, China. JAMA Oncol 2020;6:1108-10.

21. Liang W, Guan W, Chen R, et al. Cancer patients in SARS-CoV-2 infection: a nationwide analysis in China. Lancet Oncol 2020;21:335-7

22. Giannakoulis VG, Papoutsi E, Siempos II. Effect of cancer on clinical outcomes of patients with COVID-19: a meta-analysis of patient data. JCO Glob Oncol 2020;6:799-808

23. Venkatesulu BP, Chandrasekar VT, Girdhar P, et al. A systematic review and meta-analysis of cancer patients affected by a novel coronavirus. Preprint. medRxiv. 2020;2020.05.27.20115303.

24. Tian J, Yuan X, Xiao J, et al. Clinical characteristics and risk factors associated with COVID-19 disease severity in patients with cancer in Wuhan, China: a multicentre, retrospective, cohort study. Lancet Oncol 2020;21:893-903.

25. Dai M, Liu D, Liu M, et al. Patients with cancer appear more vulnerable to SARS-CoV-2: a multicenter study during the COVID-19 outbreak. Cancer Discov 2020;10:783-91.

26. Jee J, Foote MB, Lumish M, et al. Chemotherapy and COVID-19 outcomes in patients with cancer. J Clin Oncol 2020;38: 3538-46.

27. Mehta V, Goel S, Kabarriti R, et al. Case fatality rate of cancer patients with COVID-19 in a New York hospital system. Cancer Discov 2020;10:935-41.

28. Rogado J, Pangua C, Serrano-Montero G, et al. Covid-19 and lung cancer: a greater fatality rate? Lung Cancer 2020;146:19-22.

29. Conti P, Ronconi G, Caraffa A, et al. Induction of pro-inflammatory cytokines (IL-1 and IL-6) and lung inflammation by Coronavirus-19 (COVI-19 or SARS-CoV-2): anti-inflammatory strategies. J Biol Regul Homeost Agents 2020;34:327-31.

30. Zhang Y, Zhong Y, Pan L, Dong J. Treat 2019 novel coronavirus (COVID-19) with IL-6 inhibitor: are we already that far? Drug Discov Ther 2020;14:100-2.

31. Dai MY, Chen Z, Leng Y, et al. Patients with lung cancer have high susceptibility of COVID-19: a retrospective study in Wuhan, China. Cancer Control 2020;27:1073274820960467.

32. Luo P, Liu Y, Qiu L, Liu X, Liu D, Li J. Tocilizumab treatment in COVID-19: a single center experience. J Med Virol 2020;92:814-8.

33. David E, Mifthun MD. Overview of the initial treatment and prognosis of lung cancer. UptoDate. [Last accesses on 2 Feb 2021] 
34. Chen W, Zheng R, Baade PD, et al. Cancer statistics in China, 2015. CA Cancer J Clin 2016;66:115-32.

35. Chamilos G, Lionakis MS, Kontoyiannis DP. Are all patients with cancer at heightened risk for severe Coronavirus Disease 2019 (COVID-19)? Clin Infect Dis 2020; ciaa1079.

36. Zhang L, Zhu F, Xie L, et al. Clinical characteristics of COVID19-infected cancer patients: A retrospective case study in three hospitals within Wuhan, China. Ann Oncol 2020;31:894-901.

37. Kuderer NM, Choueiri TK, Shah DP, et al. Clinical impact of COVID-19 on patients with cancer (CCC19): A cohort study. Lancet 2020;395:1907-18.

38. Lee LY, Cazier JB, Angelis V, et al. COVID-19 mortality in patients with cancer on chemotherapy or other anticancer treatments: a prospective cohort study. Lancet 2020;395:1919-26.

39. ASCO special report: a guide to cancer care delivery during the COVID-19 pandemic. Available from: https://www.asco.org/sites/newwww.asco.org/files/content-files/2020-ASCO-Guide-Cancer-COVID19.pdf. [Last accesses on 2 Feb 2021]

40. Posicionamiento-recomendaciones SEOM sobre el cribado serológico previo al inicio de una quimioterapia inmunosupresora durante la pandemia COVID-19. Paciente asintomático sin infección COVID-19 conocida. Available from: https://seom.org/images/ Posicionamiento_TESTS_COVID_QUIMIO.pdf. [Last accesses on 2 Feb 2021]

41. Di Giacomo AM, Gambale E, Monterisi S, Valente M, Maio M. SARS-COV-2 infection in patients with cancer undergoing checkpoint blockade: Clinical course and outcome. Eur J Cancer 2020;133:1-3.

42. COVID-19 Provider and Practice information. Available from: https://www.asco.org/asco-coronavirus-information/provider-practicepreparedness-covid-19. [Last accesses on 2 Feb 2021]

43. Coccia M. Path-breaking target therapies for lung cancer and a far-sighted health policy to support clinical and cost effectiveness. Health Policy Technol 2014;3:74-82.

44. Zhang H, Xie C, Huang Y. Treatment and outcome of a patient with lung cancer Infected with severe acute respiratory syndrome Coronavirus-2. J Thorac Oncol 2020;15:e63-4.

45. COVID-19 rapid guideline: Delivery of radiotherapy. Available from: https://www.nice.org.uk/guidance/ng162/chapter/4-Patients-withsymptoms-of-COVID-19-at-presentation. [Last accesses on 2 Feb 2021]

46. Purandare NC, Rangarajan V. Imaging of lung cancer: implications on staging and management. Indian J Radiol Imaging 2015;25:10920.

47. Xu B, Xing Y, Peng J, et al. Chest CT for detecting COVID-19: a systematic review and meta-analysis of diagnostic accuracy. Eur Radiol 2020;30:5720-7.

48. Ai T, Yang Z, Hou H, et al. Correlation of chest CT and RT-PCR testing for Coronavirus Disease 2019 (COVID-19) in China: a report of 1014 cases. Radiology 2020;296:E32-E40.

49. Xu B, Xing Y, Peng J, et al. (2020) Chest CT for detecting COVID-19: a systematic review and meta-analysis of diagnostic accuracy. Eur Radiol 2020;30:5720-7.

50. Liu M, Zeng W, Wen Y, Zheng Y, Lv F, Xiao K. COVID-19 pneumonia: CT findings of 122 patients and differentiation from influenza pneumonia. Eur Radiol 2020;30:5463-9.

51. Shaverdian N, Shepherd AF, Rimner A, et al. Need for caution in the diagnosis of radiation pneumonitis during the COVID-19 pandemic. Adv Radiat Oncol 2020;5:617-20.

52. Nishino M, Ramaiya NH, Awad MM, et al. PD-1 inhibitor-related pneumonitis in advanced cancer patients: Radiographic patterns and clinical course. Clin Cancer Res 2016;22:6051-60.

53. Wang Y, Dong C, Hu Y, et al. Temporal changes of CT findings in 90 patients with COVID-19 pneumonia: a longitudinal study. Radiology 2020;296:E55-E64.

54. Coccia M. Deep learning technology for improving cancer care in society: new directions in cancer imaging driven by artificial intelligence. Technology in Society 2020;60:101198.

55. Patt D, Gordan L, Diaz M, et al. Impact of COVID-19 on cancer care: how the pandemic is delaying cancer diagnosis and treatment for American seniors. JCO Clin Cancer Inform 2020;4:1059-71.

56. Singh AP, Berman AT, Marmarelis ME, et al. Management of lung cancer during the COVID-19 pandemic. JCO Oncol Pract 2020;16:579-86.

57. Indini A, Aschele C, Cavanna L, et al. Reorganisation of medical oncology departments during the novel coronavirus disease-19 pandemic: a nationwide Italian survey. Eur J Cancer 2020;132:17-23.

58. Wang XS, Zeng M. Challenges for a cancer center in the Novel Coronavirus Pneumonia epidemic. Technol Cancer Res Treat 2020;19:1533033820945774.

59. Mazzone PJ, Gould MK, Arenberg DA, et al. Management of lung nodules and lung cancer screening during the COVID-19 pandemic: CHEST expert panel report. Chest 2020;158:406-15.

60. Lonergan PE, Washington Iii SL, Branagan L, et al. Rapid utilization of telehealth in a comprehensive cancer center as a response to COVID-19: cross-sectional analysis. J Med Internet Res 2020;22:e19322.

61. Oropallo A. Coronavirus disease 2019 (COVID-19): Issues related to wound care a telehealth managemen. UptoDate. [Last Accessed on 2 Feb 2021]

62. Dinesen B, Nonnecke B, Lindeman D, et al. Personalized telehealth in the future: a global research agenda. J Med Internet Res 2016;18:e53.

63. Higgison IJ, Sen-Gupta GJ. Place of care in advanced cancer: a qualitative systematic literature review of patient preferences. $J$ Palliat Med 2000;3:287-300. 
64. Cartoni C, Brunetti GA, D'Elia GM, et al. Cost analysis of a domiciliary program of supportive and palliative care for patients with hematologic malignancies. Haematologica 2007;92:666-73.

65. Centro de Coordinación de Alertas y Emergencias Sanitarias. Documento técnico: Manejo do- miciliario del COVID-19 [Online]. Madrid, España: Dirección General de Salud Pública, Calidad e Innovación, Ministerio de Sanidad; 2020 Available at: https://www.mscbs.gob.es/ profesionales/saludPublica/ccayes/alertasActual/nCov/documentos/Manejo_primaria.pdf [Last Accessed on 2 Feb 2021]

66. Wanden-Berghe C, Sanz-Valero J. La Hospitalización a Domicilio en la enfermedad del COVID-19. Hosp Domic 2020;4:5-7.

67. Tralongo P, Ferraù F, Borsellino N, et al. Cancer patient-centered home care: a new model for health care in oncology. Ther Clin Risk Manag 2011;7:387-92.

68. Depledge J. Chemotherapy in the community for adult patients with cancer. Br J Community Nurs 2012;17:214-20.

69. Murthy V, Wilson J, Suhr J, et al. Moving cancer care closer to home: a single-centre experience of home chemotherapy administration for patients with myelodysplastic syndrome. ESMO Open 2019;4:e00434.

70. Finley C, Prashad A, Camuso N, et al. Guidance for management of cancer surgery during the COVID-19 pandemic. Can J Surg 2020;63:S2-S4.

71. Kumar S, Chmura S, Robinson C, et al. Alternative multidisciplinary management options for locally advanced NSCLC during the Coronavirus Disease 2019 global pandemic. J Thorac Oncol 2020;15:1137-46.

72. Muralidhar V, Dee EC, D'Amico AV. Sequencing treatments for cancer during the COVID-19 pandemic. Am J Clin Oncol 2020;43:457-8.

73. Kutikov A, Weinberg DS, Edelman MJ, Horwitz EM, Uzzo RG, Fisher RI. A war on two fronts: cancer care in the time of COVID-19. Ann Intern Med 2020;172:756-8.

74. Xia Y, Jin R, Zhao J, Li W, Shen H. Risk of COVID-19 for patients with cancer. Lancet Oncol 2020;21:e180.

75. Salinas P, Travieso-González A, Vergara-Uzcategui CE, Macaya F, Núñez-Gily IJ, Fernández-Ortiz A. Temporal relation between invasively managed acute coronary syndromes and confinement during the current COVID-19 pandemic. REC Interv Cardiol 2020;2:307-9

76. British Columbia Cancer Agency. COVID-19 and cancer treatments-Information for patients. March 23, 2020. Available from: www. bccancer. bc.ca/about/news-stories/news/2020/covid-19-and-cancer-treatments. [Last Accessed on 2 Feb 2021]

77. Cancer Council of Australia. Information and support regarding cancer and COVID-19. Available from: https://www.cancer.org.au/ support-and-services/cancer-and-covid-19. [Last Accessed on 2 Feb 2021]

78. National Institute for Health and Care Excellence. COVID-19 rapid guideline: delivery of systemic anticancer treatments. March 20, 2020. Available from: https://www.nice.org.uk/guidance/ng161 [Last Accessed on 2 Feb 2021]

79. European Society of Medical Oncology. COVID-19 and cancer. Available from: www.esmo.org/newsroom/covid-19-and-cancer?hit=ehp. [Last Accessed on 2 Feb 2021]

80. Royal College of Radiologists. Repository of advisory documents for cancer treatment during the coronavirus (COVID-19) pandemic. Available from: https://www.rcr.ac.uk/college/coronavirus-covid-19-what-rcr-doing/clinical-information/coronavirus-covid-19-cancer [Last Accessed on 2 Feb 2021]

81. Mannelli C. Whose life to save? Scarce resources allocation in the COVID-19 outbreak. J Med Ethics 2020;46:364-6.

82. Ueda M, Martins R, Hendrie PC, et al. Managing cancer care during the COVID-19 pandemic: agility and collaboration toward a common goal. J Natl Compr Canc Netw 2020;18:1-4.

83. American College of Surgeons. Ethical framework for the allocation of resources in the event of shortages. Available from: www.facs.org/ covid-19/ ethics. [Last Accessed on 2 Feb 2021]

84. Sterpetti AV. Lessons learned during the COVID-19 virus pandemic. J Am Coll Surg 2020;230:1092-3.

85. Doherty GJ, Goksu M, de Paula BHR. Rethinking cancer clinical trials for COVID-19 and beyond. Nat Cancer 2020;29:1-5.

86. Shuman AG, Pentz RD. Cancer research ethics and COVID-19. Oncologist 2020;25:458-9.

87. Zhang WR, Wang K, Yin L, et al. Mental health and psychosocial problems of medical health workers during the COVID-19 epidemic in China. Psychother Psychosom 2020;89:242-50.

88. Demartini K, Konzen VM, Siqueira MO, et al. Care for frontline health care workers in times of COVID-19. Rev Soc Bras Med Trop 2020;53:e20200358.

89. Sykes C, Borthwick C, Baker E. Mental health and wellbeing in the medical profession. The British Medical Association 2019. Available from: https://www.bma.org.uk/media/1362/bma-mental-health-and-wellbeing-medical-profession-full-report-oct-2019.pdf. [Last Accessed on 2 Feb 2021]

90. Macía-Rodríguez C, Martín Iglesias D, Moreno Diaz J, et al. Síndrome de burnout en especialistas en medicina interna y factores asocaidos a su desarrollo. Revista Clínica Española 2020;6:331-8.

91. Chen Q, Liang M, Li Y, et al. Mental health care for medical staff in China during the COVID-19 outbreak. Lancet Psychiatry 2020;7:e15-6.

92. Greenbaum Z. Psychologist leads innovative approach to tackle psychological toll of COVID-19. Am Psychol Assoc Available from: https://www.apa.org/news/apa/2020/03/psychologist-covid-19. [Last Accessed on 2 Feb 2021]

93. Vlessides M. COVID-19: Mental health pros come to the aid of frontline comrades. Medscape 2020. Available from https://www. medscape.com/viewarticle/928004?nlid=134830_2052\&src=WNL_mdplsnews_200403_mscpedit_psyc\&uac=216237CR\&spon=12\&im $\mathrm{pID}=2334311 \& \mathrm{faf}=1$ [Last Accessed on 2 Feb 2021]

94. Forman R, Atun R, McKee M, Mossialos E. 12 Lessons learned from the management of the coronavirus pandemic. Health Policy 2020;124:577-80.

95. Coccia M. Problem-driven innovations in drug discovery: Co-evolution of the patterns of radical innovation with the evolution of problems. Health Policy Technol 2016;5:143-55. 